\title{
Voltage Sources in 2D Fourier-Based Analytical Models of Electric Machines
}

\author{
Bert Hannon, ${ }^{1,2}$ Peter Sergeant, ${ }^{1,2}$ and Luc Dupré ${ }^{2}$ \\ ${ }^{1}$ Department of ITઐC, Electrical Energy Research Group, Ghent University, V. Vaerwyckweg 1, 9000 Gent, Belgium \\ ${ }^{2}$ Department of EESA, Electrical Energy Laboratory, Ghent University, Technologiepark-Zwijnaarde 913, 9052 Gent, Belgium
}

Correspondence should be addressed to Bert Hannon; bert.hannon@ugent.be

Received 29 June 2015; Accepted 19 September 2015

Academic Editor: Jamal Berakdar

Copyright (c) 2015 Bert Hannon et al. This is an open access article distributed under the Creative Commons Attribution License, which permits unrestricted use, distribution, and reproduction in any medium, provided the original work is properly cited.

The importance of extensive optimizations during the design of electric machines entails a need for fast and accurate simulation tools. For that reason, Fourier-based analytical models have gained a lot of popularity. The problem, however, is that these models typically require a current density as input. This is in contrast with the fact that the great majority of modern drive trains are powered with the help of a pulse-width modulated voltage-source inverter. To overcome that mismatch, this paper presents a coupling of classical Fourier-based models with the equation for the terminal voltage of an electric machine, a technique that is well known in finite-element modeling but has not yet been translated to Fourier-based analytical models. Both a very general discussion of the technique and a specific example are discussed. The presented work is validated with the help of a finite-element model. A very good accuracy is obtained.

\section{Introduction}

With evermore strict demands on the performance of electric machines, the importance of optimizations during early design stages is growing. Typically, such optimizations have a very large design space. In order to limit the associated computational burden, fast machine models are required. Therefore, often very simple models are used $[1,2]$. However, typically, these models require a lot of simplifications or experimental parameters. One class of models that combines a high level of accuracy and a low computational time is the class of Fourier-based analytical models. Moreover, these Fourier-based analytical models do not require any experimental parameters. It is therefore no surprise that the interest in such models is very high [3-11].

The Fourier-based analytical models that are presented in literature require a current density as input. The problem is that nowadays most electric drives are powered with the help of a pulse-width modulated voltage-source inverter. To overcome that mismatch, this work extends the magnetic calculations of Fourier-based models with the equation for the terminal voltage of an electric machine. By doing so, it is possible to directly account for a voltage source instead of the classical approach of imposing a current density. The technique of combining magnetic calculations with the equation for the terminal voltage has already proven its worth in finite-element models [12]. However, to date, it has not yet been translated to Fourier-based analytical models. Moreover, despite its apparent simplicity, a general formulation of the technique is not obvious. For those reasons, this work presents both a general discussion and a specific example on how to couple classical Fourier-based models with the equation for the terminal voltage.

The presented work consists of three major parts.

Firstly, a very general discussion on the technique of coupling magnetic calculations with the equation for the terminal voltage of electric machines is presented. This discussion is spread over Sections 2-5. Section 2 introduces the magnetic calculations of classic Fourier-based models. In Sections 3 and 4, the equation for the terminal voltage is rewritten so that it can be used in Fourier-based analytical models. These two aspects are combined in Section 5, resulting in a model that directly accounts for the terminal voltage of electric machines.

In the second part of this work, the theoretical discussion of part one is concretized with the help of an example. 
The machine that is considered is a rotating, radial-flux permanent-magnet synchronous machine (PMSM) for highspeed applications. This machine is discussed in Section 6.

The same machine topology is used in the third part to validate the presented work with the help of a finite-element model. This is done in Section 7. Section 8 concludes the work.

\section{Fourier-Based Analytical Models}

By means of introduction, this section briefly discusses Fourier-based analytical models. The goal is to present a set of equations that defines the magnetic field as a function of the geometry, the remanent magnetic flux density, and the current density.

Typically, Fourier-based models use a potential formulation, that is, the magnetic vector potential (MVP) or the Magnetic Scalar Potential (MSP), to rewrite Maxwell's equations in the form of a second-order partial-differential equation. Because of the fact that it is the most generally valid formulation, the focus in this work is on the MVP.

The magnetic vector potential, which is denoted by $\mathbf{A}$, is defined through its curl:

$$
\mathbf{B}=\nabla \times \mathbf{A},
$$

where $\mathbf{B}$ is the magnetic flux density.

The magnetic field and therefore the MVP depend on time and space. Fourier-based models rely on periodicities in these time and spatial dependencies to formulate a solution for $\mathbf{A}$. While time is simply denoted by $t$, a coordinate system has to be chosen to describe the spatial dependency. This choice usually depends on the shape of the studied problem. For the sake of generality, an arbitrary coordinate system $(q, p, l)$ is defined so that the spatial periodicity is along the $p$-axis. The $p$-direction is referred to as the parallel direction. The $q$-direction is referred to as the normal direction and the $l$-direction is the longitudinal direction. Note that, in practice, most authors use a Cartesian coordinate system $(q=y, p=$ $x, l=z)$, a cylindrical coordinate system $(q=r, p=\varphi, l=z)$, or an axisymmetric coordinate system $(q=r, p=z, l=\varphi)$. More information on the use of these coordinate systems can be found in [6].

For simplicity, in this work, it is assumed that the studied problem is invariant along the longitudinal axis; that is, the problem can be regarded in two dimensions. This approximation is very generally used and implies that the MVP's only nonzero component is the one along the $l$-direction [3-9]. Evidently, this nonzero component is independent from the invariant direction. Mathematically, this assumption implies the following:

$$
\mathbf{A}=A(q, p, t) \cdot \mathbf{e}_{l}
$$

In rotational machines with a radial flux, for example, usually a cylindrical system with the $z$-direction along the machine's axis is used. The problem is then often assumed to be invariant along the machine's axis, that is, along the $z$-direction.
2.1. Governing Equation. As already mentioned, the MVP is used to rewrite Maxwell's equations in the form of a differential equation. Assuming quasistatic conditions, this governing equation is written as $[3,4]$

$$
\nabla^{2} \mathbf{A}-\mu \sigma \frac{\partial \mathbf{A}}{\partial t}=-\mu \mathbf{J}_{\mathrm{ext}}-\nabla \times \mathbf{B}_{\mathrm{rem}}
$$

where the time-derivative term accounts for eddy-currents and the two terms in the right-hand side of (3) account for the sources, that is, externally imposed current densities and remanent magnetic flux densities.

2.2. Subdomains. In order to simplify the governing equation, the problem is now divided in $N_{\nu}$ regions with constant electromagnetic properties, that is, constants $\mu$ and $\sigma$. Such regions are called subdomains; they are denoted with index $\nu$. Usually, the governing equation will reduce to a Laplace, a Poisson, or a Helmholtz equation in each of the subdomains.

2.3. Separation of Variables. Using separation of variables to solve (3) in subdomain $v, A^{(v)}(q, p, t)$ can be written as follows:

$$
\sum_{n=-\infty}^{\infty} \sum_{k=-\infty}^{\infty} A_{n, k}^{(v)}(q) e^{j\left(\left(2 k \pi / T_{p}\right)\left(p-p_{0}^{(\nu)}\right)-\left(2 n \pi / T_{t}\right) t\right)}
$$

with

$$
A_{n, k}^{(v)}(q)=U_{n, k}^{(v)} f_{n, k}^{(v)}(q)+V_{n, k}^{(v)} g_{n, k}^{(v)}(q)+h_{n, k}^{(v)}(q),
$$

where $n$ and $k$ are the time- and spatial-harmonic orders, $p_{0}^{(v)}$ is the starting angle of subdomain $\nu, U_{n, k}^{(\nu)}$ and $V_{n, k}^{(v)}$ are integration constants, $f_{n, k}^{(\nu)}(q)$ and $g_{n, k}^{(\nu)}(q)$ are $q$-dependent functions that are defined by the governing equation, and $T_{t}$ and $T_{p}$ are the time and spatial periods. $h_{n, k}^{(\nu)}(q)$ is the particular solution of the governing equation, which is either zero or dependent on the source terms in subdomain $\nu\left(\mathbf{J}_{\text {ext }}^{(v)}\right.$ and $\left.\mathbf{B}_{\text {rem }}^{(v)}\right)$.

Note that in practice the infinite summations have to be truncated. The highest time- and spatial-harmonic orders that are considered are $h_{n}$ and $h_{k}$, respectively.

2.4. Boundary Conditions. The next step is to link the solutions in the different subdomains back together. This is done by imposing physical boundary conditions, that is, Ampère's law and Gauss law for magnetism. On the boundary between subdomains $v$ and $v+1$, these conditions are written as follows:

$$
\begin{gathered}
\mathbf{n} \times\left(\mathbf{H}^{(\nu)}-\mathbf{H}^{(\nu+1)}\right)=\mathbf{K}^{(\nu)}, \\
\mathbf{n} \cdot\left(\mathbf{B}^{(\nu)}-\mathbf{B}^{(\nu+1)}\right)=\mathbf{0},
\end{gathered}
$$

where $\mathbf{H}^{(v)}$ is the magnetic field strength in subdomain $v$ and $\mathbf{K}^{(v)}$ is the current density on the boundary between subdomains $v$ and $v+1$. Using the definition of the MVP and the magnetic constitutive relation, these boundary conditions can be written in terms of the MVP [3-9]. 
2.5. System of Equations. In a final step, a system of equations is constructed by combining the equation for the MVP (4) with the boundary conditions (6a) and (6b). As shown in [3], the system can be solved separately for every time-harmonic order $(n)$. In matrix form, the result is then written as follows:

$$
\left[C_{n}^{1}\right] \cdot\left[X_{n}\right]=-\left[C_{n}^{2}\right] \cdot\left[J_{n}\right]+\left[C_{n}^{3}\right] \cdot\left[B_{n}\right],
$$

where $\left[X_{n}\right]$ is a $2\left(2 h_{k}+1\right) N_{v} \times 1$ matrix, every row of which refers to an integration constant. Analogously, $\left[B_{n}\right]$ is a $\left(2 h_{k}+\right.$ 1) $N_{v} \times 1$ matrix that contains all of the spatial-harmonic coefficients of the remanent magnetic induction in each of the subdomains. $\left[J_{n}\right]$ contains the current densities in each of the subdomains. Although some authors account for the spatial dependency of the current density in the subdomains $[11,13]$, usually the current density is assumed to be constant in a subdomain. For simplicity reasons, this assumption is also adopted in this work. $\left[J_{n}\right]$ is then a $N_{v} \times 1$ matrix, with each row referring to the current density in a subdomain. $\left[C_{n}^{1}\right],\left[C_{n}^{2}\right]$, and $\left[C_{n}^{3}\right]$ are coefficient matrices with respective size of $2\left(2 h_{k}+1\right) N_{v} \times 2\left(2 h_{k}+1\right) N_{v}, 2\left(2 h_{k}+1\right) N_{v} \times N_{v}$, and $2\left(2 h_{k}+1\right) N_{v} \times\left(2 h_{k}+1\right) N_{v}$. These matrices depend on the machine's geometry and are therefore not regarded here.

The above implies that the system contains $2\left(2 h_{k}+1\right) N_{v}$ unknown integration constants and an equal amount of boundary conditions.

By solving (7) for every time-harmonic order, the MVP is uniquely defined in every subdomain. This implies that the magnetic field in the studied machine is known. However, if the current densities, that is, $\left[J_{n}\right]$, are unknown, the system is underdetermined and extra $N_{v}$ equations are required. In the following sections, these equations will be derived from the equation for the terminal voltage of an electric machine.

2.6. Assumptions. The analytical approach described in the above requires some basic assumptions. Primarily, the machine is assumed to operate in steady state. This assumption is necessary in order to impose a time periodicity, as explained in Section 2. A second assumption is that the problem can be regarded in 2 dimensions. Although this assumption is not strictly necessary, it greatly simplifies the calculus. Thirdly, the situation is assumed to be quasistatic. Again, this approximation results in a reduced computational complexity. For that same reason, the externally imposed current density is assumed to be spatially constant in every current-carrying region, for example, a slot. Finally, the machine's soft-magnetic material is assumed to be infinitely permeable. This assumption is mandatory to analytically solve the governing equation.

These five assumptions are listed as follows:

(i) Steady-state operation.

(ii) $2 \mathrm{D}$ approximation of the problem.

(iii) Quasistatic situation.

(iv) Uniform current density in every subdomain.

(v) Infinite permeability of the iron.

\section{Terminal Voltage and Current Density}

The previous section introduced the calculation of the magnetic field of an electric machine with the help of the Fourierbased modeling technique. As can be seen from (3), one of the inputs of such a model is the current density. In order to input the terminal voltages instead, this section links the current densities of every subdomain to the applied terminal voltages. The discussion starts from the classical equation for the terminal voltage of an arbitrary coil $c$ in an electric machine:

$$
v^{(c)}(t)=R i^{(c)}(t)+\frac{d \psi_{\mathrm{tot}}^{(c)}(t)}{d t} .
$$

The flux coupled with a coil $c\left(\psi_{\text {tot }}^{(c)}(t)\right)$ can be divided in a component related to the active part of the coil $\left(\psi^{(c)}(t)\right)$ and a component related to the end-windings $\left(\psi_{\mathrm{ew}}^{(c)}(t)\right)$. The above then results in the following:

$$
\begin{aligned}
v^{(c)}(t) & =R i^{(c)}(t)+\frac{d \psi_{\mathrm{ew}}^{(c)}(t)}{d t}+\frac{d \psi^{(c)}(t)}{d t} \\
& =R i^{(c)}(t)+L_{\mathrm{ew}} \frac{d i^{(c)}(t)}{d t}+\frac{d \psi^{(c)}(t)}{d t} .
\end{aligned}
$$

Note that it is assumed that every coil has the same ohmic resistance $R$ and the same end-windings inductance $L_{\mathrm{ew}}$. These values can be obtained with classical formulas, such as the ones found in [14].

The functions in (9) can be written in terms of their Fourier series:

$$
\begin{aligned}
\sum_{n=-\infty}^{\infty} V_{n}^{(c)} e^{-j n \omega t}= & \sum_{n=-\infty}^{\infty} R I_{n}^{(c)} e^{-j n \omega t}+L_{\mathrm{ew}} \frac{d I_{n}^{(c)} e^{-j n \omega t}}{d t} \\
& +\frac{d \Psi_{n}^{(c)} e^{-j n \omega t}}{d t},
\end{aligned}
$$

where $\omega$ is the machine's mechanical pulsation, which equals $2 \pi / T_{t}$.

The above can be rewritten for every time-harmonic order $n$ separately:

$$
V_{n}^{(c)}=R I_{n}^{(c)}-j n \omega L_{\mathrm{ew}} I_{n}^{(c)}-j n \omega \Psi_{n}^{(c)} .
$$

Imply that the current's $n$th harmonic order can be calculated as follows:

$$
I_{n}^{(c)}=\frac{V_{n}^{(c)}+j n \omega \Psi_{n}^{(c)}}{R-j n \omega L_{\mathrm{ew}}} .
$$

The amplitude of the $n$th harmonic order of the current density in a subdomain $v$, for example, a slot, can now be calculated as follows:

$$
\begin{aligned}
J_{n}^{(v)} & =\frac{1}{S^{(v)}} \sum_{c=1}^{N_{c}} N^{(c, v)} I_{n}^{(c)} \\
& =\frac{1}{S^{(v)}} \sum_{c=1}^{N_{c}} \frac{N^{(c, v)}\left(V_{n}^{(c)}+j n \omega \Psi_{n}^{(c)}\right)}{R-j n \omega L_{\mathrm{ew}}} .
\end{aligned}
$$


In (13), $N^{(c, v)}$ is the amount of conductors that coil $c$ has in subdomain $v$ and $N_{c}$ is the amount of coils in the machine. $S^{(\nu)}$ is the surface of subdomain $\nu$. It can easily be reasoned that substitution of (13) in the governing equation will allow for directly accounting for the terminal voltage as a source. However, $\Psi_{n}^{(c)}$ has to be calculated from the magnetic field, that is, from the solution of the governing equation. This prevents a direct coupling between the calculation of the magnetic field and the equation for the terminal voltage. To overcome that problem, $\Psi_{n}^{(c)}$ is rewritten in terms of the magnetic vector potential in the following section.

\section{Flux Linkage}

The goal of this section is to express the flux related to the active part of an arbitrary coil $c$ as a function of the magnetic vector potential. In a first step, the flux coupled with a single turn of the coil is derived; in a second step, the flux coupled with the entire coil is regarded.

4.1. Flux Coupled with a Single Turn. The physical flux through a single turn $\tau$ of coil $c$ is calculated as the integration of the flux density over a surface spanned by that turn:

$$
\phi^{(\tau)}(t)=\iint_{S_{\tau}} \mathbf{B} \cdot d \mathbf{a}=\oint_{C_{\tau}} \mathbf{A} \cdot d \mathbf{s},
$$

where the definition of the MVP and Stokes theorem were used. $S_{\tau}$ is the surface of the turn and $C_{\tau}$ is the boundary of that surface.

Since the magnetic vector potential is assumed to only have an $l$-component, the integration of $\mathbf{A}$ along the turn's contour will only be nonzero along the $l$-direction. Noting that the MVP is independent of $l$, this implies that the integration along the contour of the turn can be rewritten as follows:

$$
\phi^{(\tau)}(t)=l_{s}\left(A^{\left(v^{+}\right)}\left(q_{\tau^{+}}, p_{\tau^{+}}, t\right)-A^{\left(v^{-}\right)}\left(q_{\tau^{-}}, p_{\tau^{-}}, t\right)\right),
$$

where $l_{s}$ is the longitudinal length of the studied machine. $v^{+}$ is the subdomain in which the direction of the integration is along the positive $l$-axis; $\mathbf{A}$ and $d \mathbf{s}$ then have the same direction and sense. $v^{-}$represents the subdomain in which the coil returns; the integration direction is opposed to the $l$-axis and thus to $\mathbf{A}$.

$q_{\tau^{+}}$and $q_{\tau^{-}}$are the normal positions of turn $\tau$ and $p_{\tau^{+}}$and $p_{\tau^{-}}$are the tangential positions of turn $\tau$.

The direction of the integration is chosen so that it corresponds to the reference direction of the current. This implies that the going conductor of turn $\tau$ is located in subdomain $v^{+}$and the returning conductor is located in subdomain $\nu^{-}$.

Equation (15) can now be rewritten as follows:

$$
\phi^{(\tau)}(t)=\sum_{\nu=1}^{N_{v}} w_{\tau}^{(v)} l_{s} A^{(v)}\left(q_{\tau}^{(v)}, p_{\tau}^{(\nu)}, t\right),
$$

where $w_{\tau}^{(v)}$ is 1 in the subdomain that contains the going conductor of $\tau,-1$ in the subdomain that contains the returning conductor, and 0 in the other subdomains.
Usually, the exact position of the turn cannot be determined; that is, $\left(q_{\tau}^{(v)}, p_{\tau}^{(v)}\right)$ is unknown. For that reason, most authors either choose an arbitrary position or use the average MVP in the considered subdomain. Doing so results in one MVP value for every subdomain; this value will be referred to as $\alpha^{(\nu)}(t)$ in the following. The flux coupled with a single turn $\tau$ of coil $c$ can then be written as follows:

$$
\phi^{(\tau)}(t)=\sum_{\nu=1}^{N_{\nu}} w_{\tau}^{(\nu)} l_{s} \alpha^{(\nu)}(t) .
$$

4.2. Flux Coupled with a Coil. The flux coupled with coil $c$ is calculated by summing the fluxes coupled with each of its turns. From (17), it can be written that

$$
\psi^{(c)}(t)=\sum_{\nu=1}^{N_{\nu}} l_{s} W^{(c, v)} \alpha^{(\nu)}(t),
$$

where

$$
W^{(c, v)}=\sum_{\tau} w_{\tau}^{(\nu)}
$$

which implies that $W^{(c, v)}$ equals $N^{(c, v)}$ if subdomain $v$ contains going conductors of $c, W^{(c, \nu)}$ equals $-N^{(c, \nu)}$ if subdomain $\nu$ contains returning conductors of $c$, and $W^{(c, v)}$ equals 0 if subdomain $v$ does not contain any conductors of coil $c$.

The $n$th time-harmonic coefficient of $\psi(t)^{(c)}$ can now be written as follows:

$$
\Psi_{n}^{(c)}=\sum_{\nu=1}^{N_{v}} l_{s} W^{(c, v)} \alpha_{n}^{(v)},
$$

where $\alpha_{n}^{(\nu)}$ is the $n$th time-harmonic coefficient of $\alpha^{(\nu)}(t)$.

Since $\alpha^{(v)}(t)$ is a direct function of the MVP in subdomain $v$, which in turn is determined by the integration constants and the source terms, (20) can be written in matrix form as follows:

$$
\begin{aligned}
\Psi_{n}^{(c)}= & l_{s}\left[W^{(c)}\right] \\
& \cdot\left(\left[C_{n}^{4}\right] \cdot\left[X_{n}\right]+\left[C_{n}^{5}\right] \cdot\left[J_{n}\right]+\left[C_{n}^{6}\right] \cdot\left[B_{n}\right]\right),
\end{aligned}
$$

where $\left[W^{(c)}\right]$ is a $1 \times N_{v}$ matrix describing the winding configuration of coil $c$; that is, $\left[W^{(c)}\right]_{1, v}=W^{(c, v)} .\left[C_{n}^{4}\right],\left[C_{n}^{5}\right]$, and $\left[C_{n}^{6}\right]$ are coefficient matrices with respective sizes of $N_{v} \times$ $2\left(2 h_{k}+1\right) N_{v}, N_{v} \times N_{v}$, and $N_{v} \times\left(2 h_{k}+1\right) N_{v}$. The content of $\left[C_{n}^{4}\right],\left[C_{n}^{5}\right]$, and $\left[C_{n}^{6}\right]$ greatly depends on the studied geometry. For that reason, it will not be regarded here. However, $\left[C_{n}^{4}\right]$, $\left[C_{n}^{5}\right]$, and $\left[C_{n}^{6}\right]$ can directly be derived from the MVP.

It can easily be seen that the above effectively expresses the flux linkage of coil $c$ in terms of the machine's geometry, the integration constant, and the classical source terms $\left(\mathbf{J}_{\text {ext }}\right.$ and $\left.\mathbf{B}_{\text {rem }}\right)$.

\section{A New System of Equations}

In (7), the system of a traditional Fourier-based analytical model is introduced. However, as mentioned in Section 2, 
that system is underdetermined if the current densities are not known. In Section 3, an equation for the current density was proposed (13); combining this equation with the equation for the flux coupled with a coil (20) gives the following:

$$
J_{n}^{(v)}=\sum_{c=1}^{N_{c}} \frac{N^{(c, v)}\left(V_{n}^{(c)}+j n \omega \sum_{i_{\nu}=1}^{N_{\nu}} l_{s} W^{\left(c, i_{\nu}\right)} \alpha_{n}^{\left(i_{\nu}\right)}\right)}{S^{(\nu)}\left(R-j n \omega L_{\mathrm{eW}}\right)} .
$$

Considering (21), this can be written in matrix notation as follows:

$$
\begin{aligned}
J_{n}^{(v)}= & {\left[M^{(\nu)}\right] \cdot\left[V_{n}\right]+j n \omega l_{s}\left[M^{(v)}\right] \cdot[W] } \\
& \cdot\left(\left[C_{n}^{4}\right] \cdot\left[X_{n}\right]+\left[C_{n}^{5}\right] \cdot\left[B_{n}\right]+\left[C_{n}^{6}\right] \cdot\left[J_{n}\right]\right),
\end{aligned}
$$

where $\left[V_{n}\right]$ is $N_{c} \times 1$ matrix containing the terminal voltages of every coil. $[W]$ is the $N_{c} \times N_{v}$ winding matrix of the machine. This means that $[W]_{c, v}=W^{(c, v)} \cdot\left[M^{(v)}\right]$ in turn is a $1 \times N_{c}$ matrix, the $c$ th element of which is calculated as follows:

$$
\left[M^{(v)}\right]_{1, c}=\frac{N^{(c, v)}}{S^{(v)}\left(R-j n \omega L_{\mathrm{ew}}\right)} .
$$

The above equation for the current density is valid in each of the $N_{v}$ subdomains. A matrix notation for the resulting set of equations can be found:

$$
\begin{aligned}
{\left[J_{n}\right]=} & {[M] \cdot\left[V_{n}\right]+j n \omega l_{s}[M] \cdot[W] } \\
& \cdot\left(\left[C_{n}^{4}\right] \cdot\left[X_{n}\right]+\left[C_{n}^{5}\right] \cdot\left[J_{n}\right]+\left[C_{n}^{6}\right] \cdot\left[B_{n}\right]\right),
\end{aligned}
$$

where $[M]$ is $N_{v} \times N_{c}$ matrix whose $v$ th row equals $\left[M^{(v)}\right]$. Rearranging gives the following:

$$
\begin{aligned}
& {\left[C_{n}^{7}\right] \cdot\left[X_{n}\right]+\left[C_{n}^{8}\right] \cdot\left[J_{n}\right]} \\
& \quad=\left[C_{n}^{9}\right] \cdot\left[B_{n}\right]+\left[C_{n}^{10}\right] \cdot\left[V_{n}\right],
\end{aligned}
$$

where $\left[C_{n}^{7}\right],\left[C_{n}^{8}\right],\left[C_{n}^{9}\right]$, and $\left[C_{n}^{10}\right]$ are matrices with respective sizes of $N_{v} \times 2\left(2 h_{k}+1\right) N_{v}, N_{v} \times N_{v}, N_{v} \times\left(2 h_{k}+1\right) N_{v}$, and $N_{v} \times N_{c}$. They are calculated as follows:

$$
\begin{aligned}
& {\left[C_{n}^{7}\right]=-j n \omega l_{s}[M] \cdot[W] \cdot\left[C_{n}^{4}\right],} \\
& {\left[C_{n}^{8}\right]=I_{N_{\nu}}-j n \omega l_{s}[M] \cdot[W] \cdot\left[C_{n}^{5}\right],} \\
& {\left[C_{n}^{9}\right]=j n \omega l_{s}[M] \cdot[W] \cdot\left[C_{n}^{6}\right],} \\
& {\left[C_{n}^{10}\right]=[M],}
\end{aligned}
$$

where $I_{N}$ is the identity matrix of size $N_{v}$.

The above implies that the combination of (7) and (26) is a system of equations that uniquely defines both the integration constants and the current densities in each of the subdomains. This system is written in matrix form as follows:

$$
\begin{aligned}
& {\left[\begin{array}{ll}
{\left[C_{n}^{1}\right]} & {\left[C_{n}^{2}\right]} \\
{\left[C_{n}^{7}\right]} & {\left[C_{n}^{8}\right]}
\end{array}\right] \cdot\left[\begin{array}{c}
{\left[X_{n}\right]} \\
{\left[J_{n}\right]}
\end{array}\right]} \\
& =\left[\begin{array}{c}
{\left[C_{n}^{3}\right] \cdot\left[B_{n}\right]} \\
{\left[C_{n}^{9}\right] \cdot\left[B_{n}\right]+\left[C_{n}^{10}\right] \cdot\left[V_{n}\right]}
\end{array}\right] .
\end{aligned}
$$

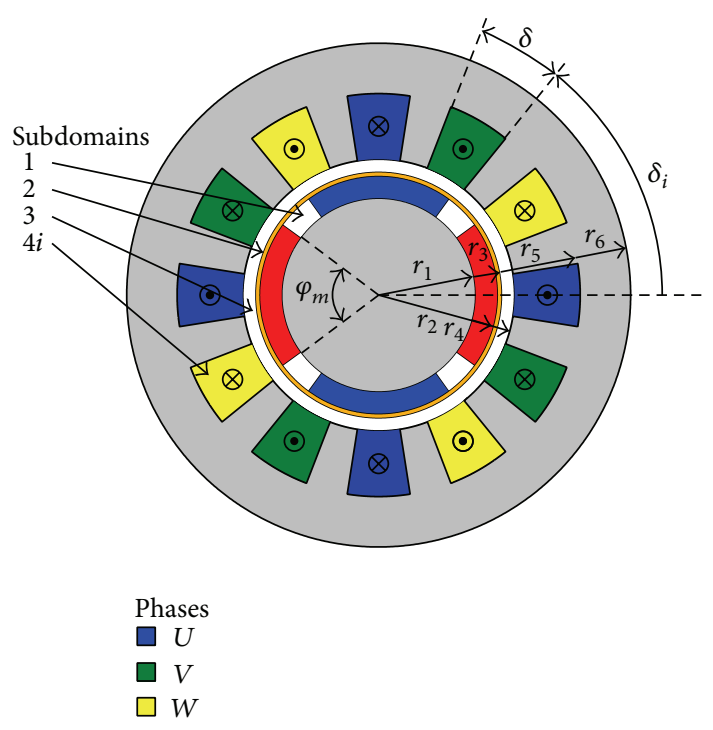

FIGURE 1: Geometry and subdomains of the studied machine.

Solving this system for every time-harmonic order will uniquely define the MVP and the current density in each of the subdomains. It can readily be seen that (28) offers to directly impose a voltage signal to the coils instead of the classical approach of imposing a current density to the subdomains.

\section{Example}

By means of example, this section discusses the determination of the coefficient matrices for a permanent-magnet synchronous machine. The goal is to clarify the above theory. The same PMSM topology will be used in Section 7 to validate the presented work.

6.1. Geometry. The machine that is studied in this section is a high-speed, radial-flux PMSM with a Shielding Cylinder (SC). The latter is a conductive sleeve that is wrapped around the magnets. Shielding Cylinders are often used in high-speed PMSMs to reduce the rotor eddy-current losses, thereby reducing the risk of permanent demagnetization of the magnets.

The studied machine is shown in Figure 1. Its geometrical parameters include outer radius of the rotor yoke $\left(r_{1}\right)$, outer radius of the magnets $\left(r_{2}\right)$, outer radius of the SC $\left(r_{3}\right)$, outer radius of the air gap $\left(r_{4}\right)$, outer radius of the slots $\left(r_{5}\right)$, and outer radius of the stator yoke $\left(r_{6}\right)$. Each magnet spans an angle of $\varphi_{m}$ radians. The slots, which are indicated with a slot number $i$, have a starting angle $\delta_{i}$ and an opening angle $\delta$. The machine has a three-phase, single-layer, and distributed winding with one slot per pole and per phase. Every phase consists of 2 parallel coils, which in turn consist of $N_{t}$ turns.

As already mentioned, the studied geometry is divided into a number of subdomains. Those subdomains are shown in Figure 1. Note that every slot is a separate subdomain, indicated with an index $4 i$ where $i$ is the slot number. 


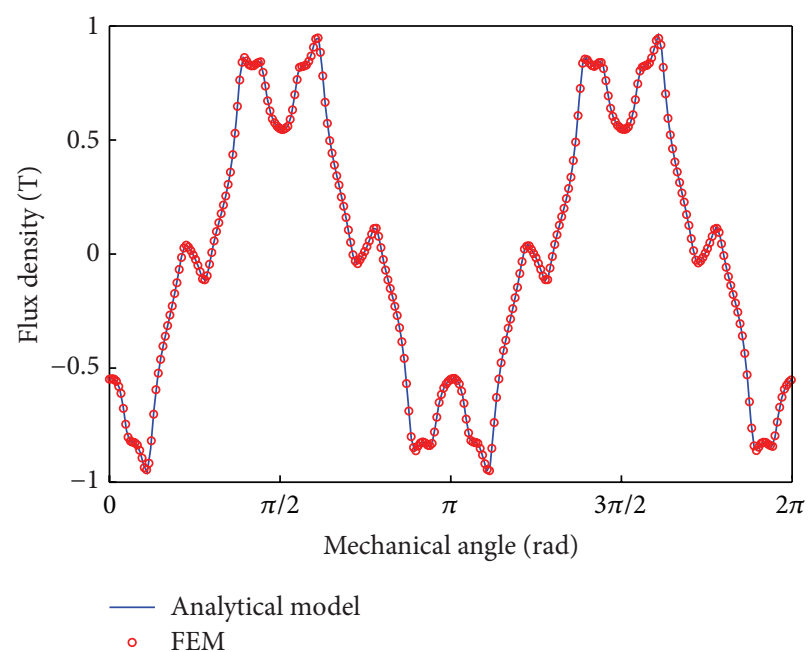

(a) Radial component

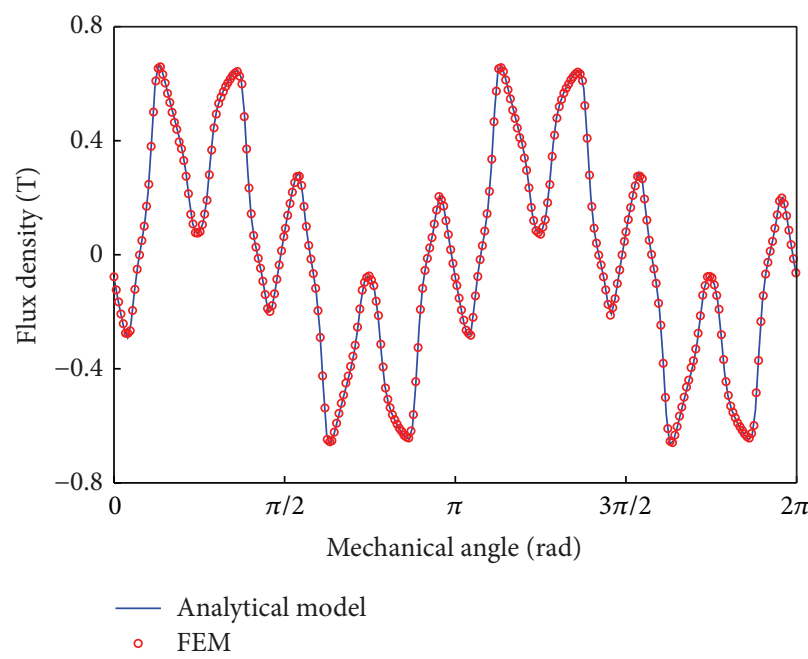

(b) Tangential component

FIGURE 2: Analytical and finite-element results for the magnetic flux density in the middle of the air gap at armature-reaction conditions.

Evidently, the machine's 6 coils are distributed solely over the slots. The externally imposed current densities in subdomains 1,2, and 3 are thus a priori known; they are zero. This means that the dimension of $\left[J_{n}\right]$ is reduced to the amount of current-carrying subdomains, that is, the 12 slots in this example. Moreover, the dimension of the electric problem (26) is reduced to the 12 slots as well.

6.2. Magnetic Calculations. A Fourier-based analytical model for the machine depicted in Figure 1 was introduced by the authors in [3]. Using a cylindrical coordinate system fixed to the rotor $(r, \varphi, t)$, these authors presented solutions for the MVP and a set of boundary conditions. This implies that the results of [3] allow for easily determining $\left[C_{n}^{1}\right],\left[C_{n}^{2}\right]$, and $\left[C_{n}^{3}\right]$. For that reason, the determination of these matrices will not be discussed in detail here.

6.3. Electric Calculations. The goal is now to determine matrices $\left[C_{n}^{7}\right],\left[C_{n}^{8}\right],\left[C_{n}^{9}\right]$, and $\left[C_{n}^{10}\right]$ from (27a), (27b), (27c), and $(27 \mathrm{~d})$ and [3]. In order to determine these matrices, $[M]$, $[W],\left[C_{n}^{4}\right],\left[C_{n}^{5}\right]$, and $\left[C_{n}^{6}\right]$ have to be calculated.

Both $[M]$ and $[W]$ strongly depend on the winding configuration of the machine; that is, they are dependent on $N^{(c, v)}$. In this example, $N^{(c, v)}$ equals $N_{t}$ if coil $c$ has conductors in subdomain $v$ and 0 if coil $c$ has no conductors in subdomain $v$. From Figure 1, the winding matrix $[W]$ can easily be determined. Its first row, which corresponds to the first coil of phase $U$, is as follows:

$$
\left[\begin{array}{llllllllllll}
N_{t} & 0 & 0 & -N_{t} & 0 & 0 & 0 & 0 & 0 & 0 & 0 & 0
\end{array}\right]
$$

where every column represents a slot, the first one being the rightmost slot of Figure 1.
Similarly, $[M]$ can be obtained from Figure 1 and (24). Its first row, corresponding to the rightmost slot in Figure 1, is as follows:

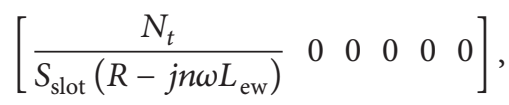

where $S_{\text {slot }}$ is the surface of a slot and every column corresponds to a coil, the first one being the first coil of phase $U$. Note that (29) and (30) regard all 12 slots and all 6 coils. Because of the machine's periodicity, it is possible to only consider 6 slots and 3 coils. This of course reduces the amount of unknowns. However, for the sake of generality, all 12 slots and all 6 coils are regarded here.

From (20) and (21), it can be seen that matrices $\left[C_{n}^{4}\right],\left[C_{n}^{5}\right]$, and $\left[C_{n}^{6}\right]$ are determined by $\alpha^{(\nu)}(t)$, that is, by the expression for the MVP in each of the subdomains. In [3], an equation for the MVP in slot $i$ was calculated as follows:

$$
\begin{aligned}
& A_{n, k}^{(4 i)}(r, \varphi, t) \\
& =\sum_{n=-\infty}^{\infty} \sum_{k=-\infty}^{\infty} A_{n, k}^{(4 i)}(r) e^{j\left((k \pi / \delta)\left(\varphi-\delta_{i}\right)+(k \pi / \delta-n) \omega t+(k \pi / \delta) \varphi_{0}\right)},
\end{aligned}
$$

where

$$
\begin{aligned}
& A_{n, k}^{(4 i)}(r) \\
& = \begin{cases}U_{n, 0}^{(4 i)}+\frac{\mu_{0} J_{n}^{(4 i)}}{2}\left(r_{5}^{2} \ln \frac{r}{r_{4}}-\frac{r^{2}-r_{4}^{2}}{2}\right), & \text { if } k=0, \\
U_{n,|k|}^{(4 i)} \frac{\left(r / r_{5}\right)^{|k \pi / \delta|}+\left(r / r_{5}\right)^{-|k \pi / \delta|}}{\left(r_{4} / r_{5}\right)^{|k \pi / \delta|}+\left(r_{4} / r_{5}\right)^{-|k \pi / \delta|}}, & \text { else. }\end{cases}
\end{aligned}
$$

As mentioned in Section 4, there are several ways to compute $\alpha^{(\nu)}(t)$, that is, to approximate $A^{(\nu)}\left(q_{\tau}^{(v)}, p_{\tau}^{(v)}, t\right)$. In this work, 
an average value is computed by integrating the MVP over the slot and dividing the result by the surface of the slot:

$$
\begin{aligned}
\alpha^{(4 i)}(t) & =\frac{1}{S_{\text {slot }}} \int_{r_{4}}^{r_{5}} \int_{\delta_{i}^{t}}^{\delta_{i}^{t}+\delta} r A^{(4 i)}(r, \varphi, t) d \varphi d r \\
& =\sum_{n=-\infty}^{\infty}\left(U_{n, 0}^{(4 i)}+\chi J_{n}^{(4 i)}\right) e^{-j n \omega_{m} t},
\end{aligned}
$$

where

$$
\chi=\frac{\mu_{0}}{8}\left(4 r_{5}^{4} \frac{\ln r_{5}-\ln r_{4}}{r_{5}^{2}-r_{4}^{2}}-3 r_{5}^{2}+r_{4}^{2}\right),
$$

which implies that the $n$th time-harmonic order of $\alpha^{(4 i)}(t)$ can be calculated as follows:

$$
\alpha_{n}^{(4 i)}=U_{n, 0}^{(4 i)}+\chi J_{n}^{(4 i)} .
$$

Combining the above with (20) and (21), it can be concluded that every row $i$ of $\left[C_{n}^{4}\right]$ contains only one nonzero element, that is, the element corresponding to $U_{n, 0}^{(4 i)}$. This element simply equals 1 . $\left[C_{n}^{5}\right]$ turns out to be a scalar matrix of size 12 , whose scalar is $\chi \cdot\left[C_{n}^{6}\right]$ is a zero matrix. The latter could indeed be expected as there is no remanent magnetic flux density in the slots.

As $[M],[W],\left[C_{n}^{4}\right],\left[C_{n}^{5}\right]$, and $\left[C_{n}^{6}\right]$ are known, $\left[C_{n}^{7}\right],\left[C_{n}^{8}\right]$, and $\left[C_{n}^{10}\right]$ can easily be calculated from (27a), (27b), (27c), and $(27 \mathrm{~d})$. This implies that the complete electromagnetical system (28) is now defined in terms of the machine's geometrical parameters, its winding distribution, and its mechanical pulsation.

\section{Validation}

The goal of this section is to validate the work that was presented in Sections 2-6. To do so, a voltage-powered PMSM, as the one presented in Figure 1, is studied with the Fourier-based analytical model. The results are compared to results obtained from a finite-element model.

The parameters of the studied machine are shown in Table 1.

A sinusoidal voltage with an amplitude of $150 \mathrm{~V}$ and a frequency of $1000 \mathrm{~Hz}$ is applied to the machine. Because of this very high frequency, Litz wire is used for the windings. This implies that the ohmic resistance of the coils can be computed with the help of Pouillet's law. The inductance of the endwindings was calculated using the formulas presented in the book of Pyrhönen et al. [14]. The highest time- and spatialharmonic orders are 50; that is, $h_{n}=h_{k}=50$.

Note that the interest here is to study the accuracy of the armature-reaction field. Indeed, as saturation is neglected, applying a voltage instead of a current has an effect on the armature-reaction situation, not on the no-load situation. For that reason, the remanent magnetic flux of the magnets $\left(\mathbf{B}_{\text {rem }}\right)$ is set to $0 \mathrm{~T}$.

The calculated radial and tangential components of $\mathbf{B}$ in the middle of the air gap are shown in Figures 2(a) and 2(b),
TABLE 1: Parameters of the studied machine.

\begin{tabular}{lcc}
\hline Symbol & Parameter & Value \\
\hline$r_{1}$ & Rotor yoke outer radius & $14.5 \mathrm{~mm}$ \\
$r_{2}$ & PM outer radius & $18.0 \mathrm{~mm}$ \\
$r_{3}$ & SC outer radius & $18.5 \mathrm{~mm}$ \\
$r_{4}$ & Air gap outer radius & $20.5 \mathrm{~mm}$ \\
$r_{5}$ & Slot outer radius & $30.4 \mathrm{~mm}$ \\
$r_{6}$ & Machine outer radius & $38.0 \mathrm{~mm}$ \\
$l_{s}$ & Stack length & $200.0 \mathrm{~mm}$ \\
$N_{s}$ & Number of slots & 12 \\
$\delta$ & Slot opening angle & $\left(\pi / N_{s}\right) \mathrm{rad}$ \\
$B_{\text {rem }}$ & PM remanent flux density & $0 \mathrm{~T}$ \\
$p$ & Number of pole pairs & 2 \\
$\varphi_{m}$ & Magnet span & $0.8(\pi / p) \mathrm{rad}$ \\
$N_{t}$ & Number of turns per coil & 5 \\
$m$ & Number of phases & 3 \\
$\sigma_{\mathrm{SC}}$ & SC conductivity & $5.96 \cdot 10^{7} \Omega \mathrm{m}$ \\
$R$ & Ohmic resistance of the coils & $0.0106 \Omega$ \\
$L_{\mathrm{ew}}$ & Inductance of the end-windings & $1.25 \cdot 10^{-6} \mathrm{H}$ \\
\hline
\end{tabular}

respectively. The agreement between the results obtained from the analytical model and the results obtained from the finite-element model is very good. This proves that the presented theory is indeed effective.

\section{Conclusion}

This work presents a technique to directly impose the terminal voltage in Fourier-based analytical models for electric machines. The idea is to combine the magnetic calculations of classical Fourier-based analytical models with the equation for the terminal voltage of an electric machine, a technique that is well known in finite-element modeling but was not yet translated to Fourier-based analytical models. Firstly, a very general discussion on the technique and its implementation is presented. This discussion is then illustrated by means of an example. Finally, the work is validated with the help of a finite-element model. The accuracy was found to be very good.

It can be concluded that this work provides an extension to the existing Fourier-based analytical models for electric machines. It presents both a very general discussion and an example on how to directly account for a voltage source instead of having to impose current sources. As the great majority of modern drive trains are powered with a voltage source, the work offers more realistic analytical modeling of electric machines. This conclusion is of great significance for machine designers who require fast and accurate modeling tools to cope with large design spaces.

Fourier-based models are a great tool because of their accurate and fast calculations. However, they are relatively complex when compared to more traditional analytical models. Although the work presented in this paper allows for even better optimization procedures, it adds to the models complexity. For that reason, machine designers should make 
a well-considered choice between a more realistic model with voltage sources or a simpler model with current sources. It would therefore be interesting to compare both approaches in future work.

\section{Conflict of Interests}

The authors declare that there is no conflict of interests regarding the publication of this paper.

\section{References}

[1] Y. Zou, D.-G. Li, and X.-S. Hu, "Optimal sizing and control strategy design for heavy hybrid electric truck," Mathematical Problems in Engineering, vol. 2012, Article ID 404073, 15 pages, 2012.

[2] Y. Wang and D. Sun, "Powertrain matching and optimization of dual-motor hybrid driving system for electric vehicle based on quantum genetic intelligent algorithm," Discrete Dynamics in Nature and Society, vol. 2014, Article ID 956521, 11 pages, 2014.

[3] B. Hannon, P. Sergeant, and L. Dupré, "2-D analytical subdomain model of a slotted PMSM with shielding cylinder," IEEE Transactions on Magnetics, vol. 50, no. 7, 2014.

[4] S. R. Holm, H. Polinder, and J. A. Ferreira, "Analytical modeling of a permanent-magnet synchronous machine in a flywheel," IEEE Transactions on Magnetics, vol. 43, no. 5, pp. 1955-1967, 2007.

[5] F. Dubas and C. Espanet, "Analytical solution of the magnetic field in permanent-magnet motors taking into account slotting effect: no-load vector potential and flux density calculation," IEEE Transactions on Magnetics, vol. 45, no. 5, pp. 2097-2109, 2009.

[6] B. L. J. Gysen, K. J. Meessen, J. J. H. Paulides, and E. A. Lomonova, "General formulation of the electromagnetic field distribution in machines and devices using fourier analysis," IEEE Transactions on Magnetics, vol. 46, no. 1, pp. 39-52, 2010.

[7] Z. Q. Zhu, L. J. Wu, and Z. P. Xia, "An accurate subdomain model for magnetic field computation in slotted surface-mounted permanent-magnet machines," IEEE Transactions on Magnetics, vol. 46, no. 4, pp. 1100-1115, 2010.

[8] T. Lubin, S. Mezani, and A. Rezzoug, "2-D exact analytical model for surface-mounted permanent-magnet motors with semi-closed slots," IEEE Transactions on Magnetics, vol. 47, no. 2, pp. 479-492, 2011.

[9] K. Boughrara, T. Lubin, and R. Ibtiouen, "General subdomain model for predicting magnetic field in internal and external rotor multiphase flux-switching machines topologies," IEEE Transactions on Magnetics, vol. 49, no. 10, pp. 5310-5325, 2013.

[10] J. Soulard and F. Meier, "Design guidelines and models for PMSMs with non-overlapping concentrated windings," COMPEL, vol. 30, no. 1, pp. 72-83, 2011.

[11] B. Chikouche, K. Boughrara, and R. Ibtiouen, "Cogging torque minimization of surface-mounted permanent magnet synchronous machines using hybrid magnet shapes," Progress in Electromagnetics Research B, vol. 62, pp. 49-61, 2015.

[12] S. Eriksson, H. Bernhoff, and M. Leijon, "A $225 \mathrm{~kW}$ direct driven PM generator adapted to a vertical axis wind turbine," Advances in Power Electronics, vol. 2011, Article ID 239061, 7 pages, 2011.

[13] L. J. Wu, Z. Q. Zhu, D. Staton, M. Popescu, and D. Hawkins, "Analytical modeling and analysis of open-circuit magnet loss in surface-mounted permanent-magnet machines," IEEE Transactions on Magnetics, vol. 48, no. 3, pp. 1234-1247, 2012.

[14] J. Pyrhönen, T. Jokinen, and V. Hrabovcová, Design of Rotating Electrical Machines, John Wiley \& Sons, Chichester, UK, 2008. 


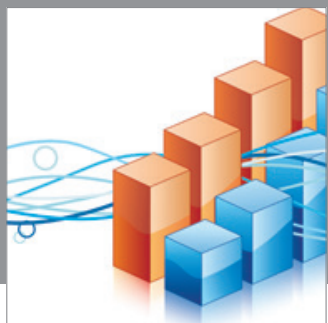

Advances in

Operations Research

mansans

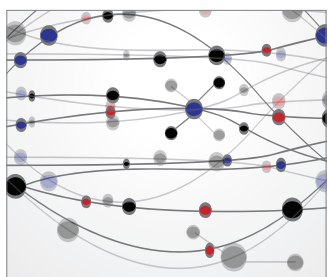

The Scientific World Journal
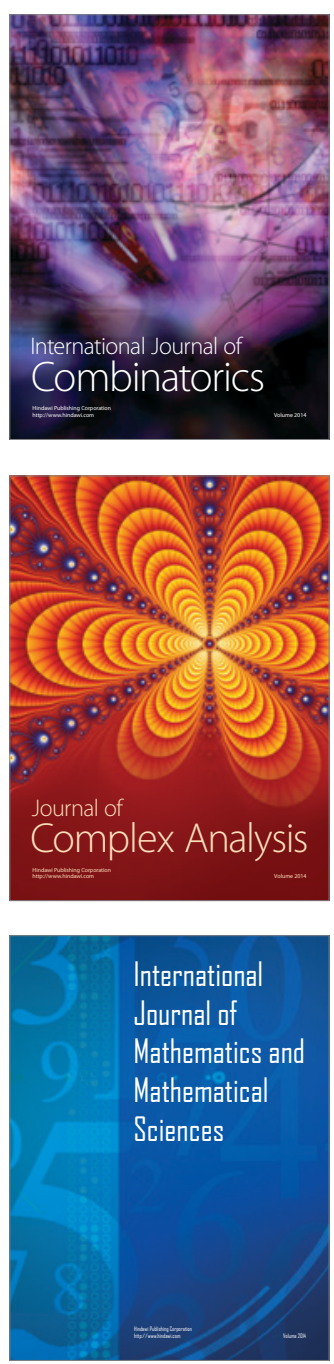
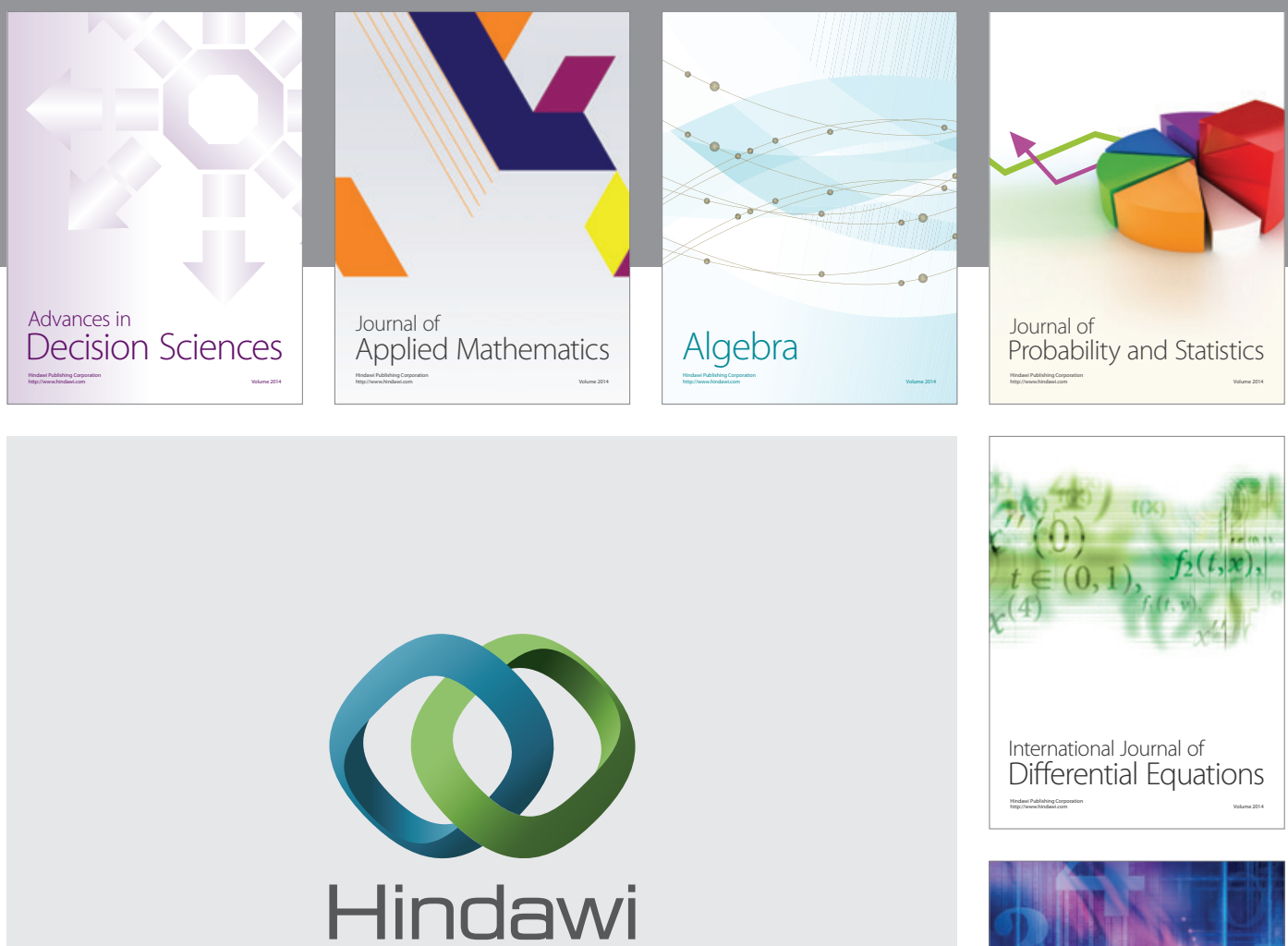

Submit your manuscripts at http://www.hindawi.com
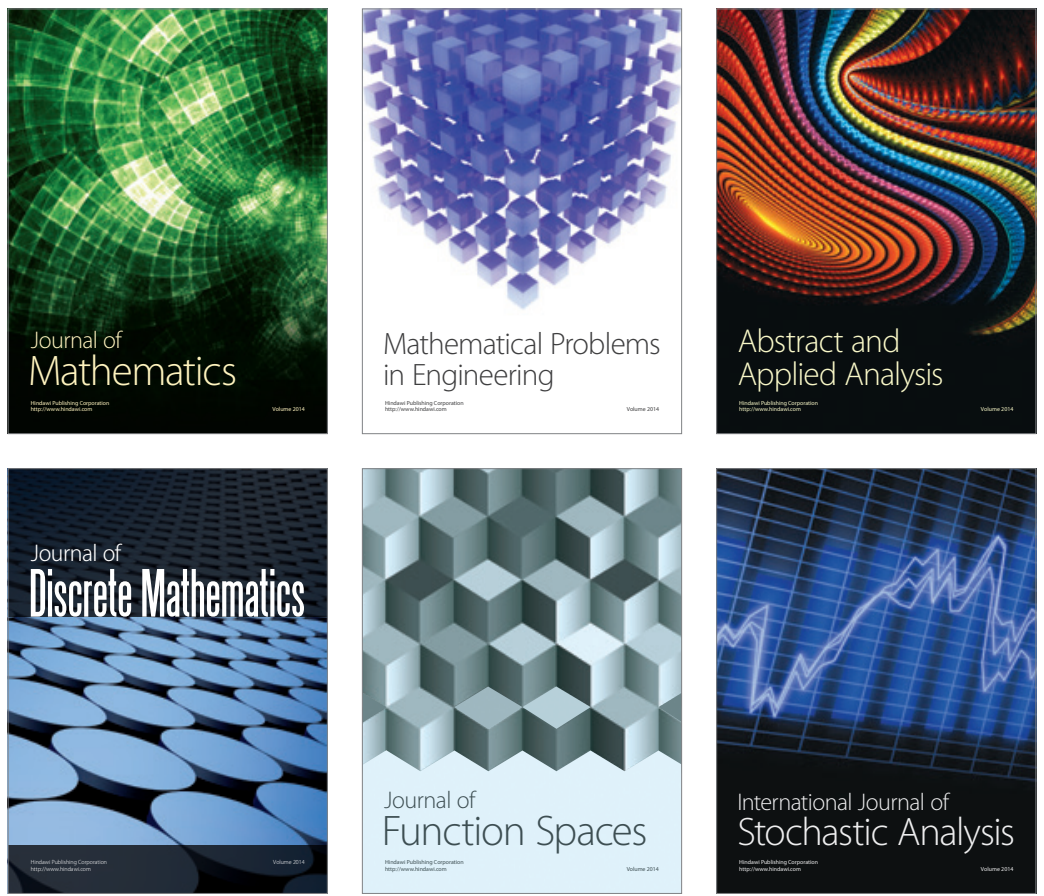

Journal of

Function Spaces

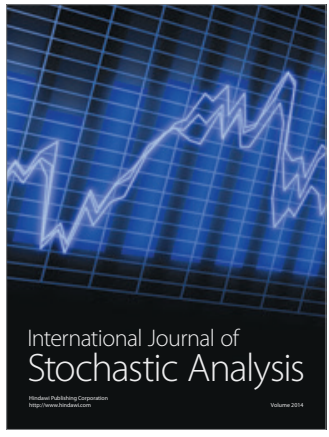

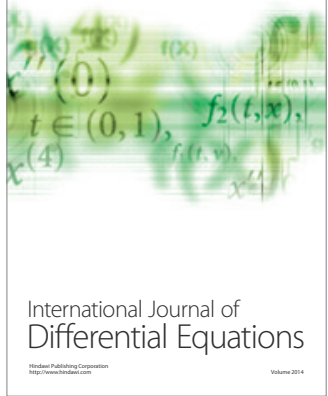
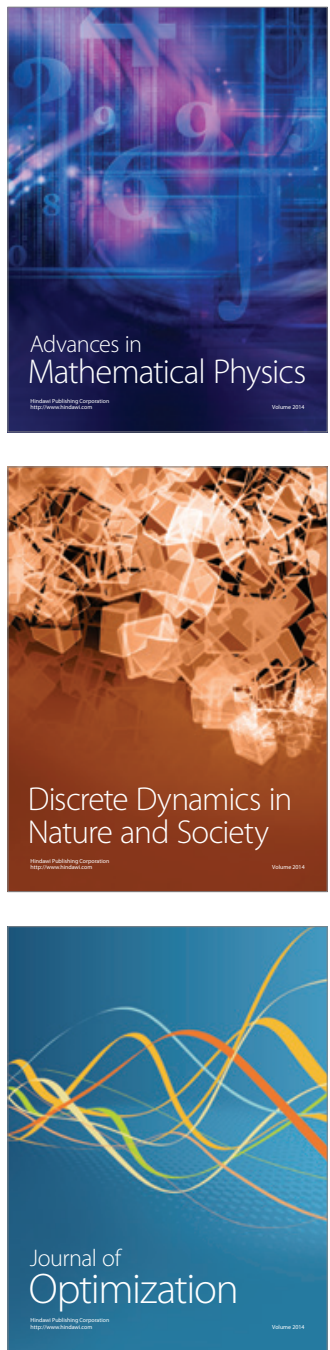